\title{
On the Conservative Finite Difference Scheme for the Generalized Novikov Equation
}

\author{
Wenxia Chen*, Qianqian Zhu, Ping Yang \\ Nonlinear Scientific Research Center, Jiangsu University, Zhenjiang, China \\ Email: *chenwx@ujs.edu.cn
}

How to cite this paper: Chen, W.X., Zhu, Q.Q. and Yang, P. (2017) On the Conservative Finite Difference Scheme for the Generalized Novikov Equation. Journal of Applied Mathematics and Physics, 5, 1776-1790.

https://doi.org/10.4236/jamp.2017.59150

Received: August 14, 2017

Accepted: September 19, 2017

Published: September 22, 2017

Copyright $\odot 2017$ by authors and Scientific Research Publishing Inc. This work is licensed under the Creative Commons Attribution International License (CC BY 4.0).

http://creativecommons.org/licenses/by/4.0/

\begin{abstract}
In this paper, we investigate a numerical method for the generalized Novikov equation. We propose a conservative finite difference scheme and use Brouwer fixed point theorem to obtain the existence of the solution of the corresponding difference equation. We also prove the convergence and stability of the solution by using the discrete energy method. Moreover, we obtain the truncation error of the difference scheme which is $R_{j}^{n}=O\left(\tau^{2}+h^{2}\right)$.
\end{abstract}

\section{Keywords}

Generalized Novikov Equation, Finite Difference Scheme, Conservation Law, Stability, Convergence

\section{Introduction}

The Camassa-Holm (CH) equation

$$
m_{t}+2 u_{x} m+u m_{x}=0
$$

was derived by Camassa and Holm [1] as a model for the unidirectional propagation of the shallow water waves over a flat bottom [2] [3], where $m=u-u_{x x}$. It was found earlier by Fuchssteiner and Fokas [4] using the recursion operator of the $\mathrm{KdV}$ equation. More interestingly, the $\mathrm{CH}$ equation could also be obtained by the tri-Hamiltonian duality approach from the bi-Hamiltonian structure of the $\mathrm{KdV}$ equation [5] [6]. So the $\mathrm{CH}$ equation could be regarded as a dual system of the $\mathrm{KdV}$ equation. The $\mathrm{CH}$ equation had attracted much attention because of its nice features: existence of peaked solitons [1] [7] (which has a discontinuous first derivative at the spike), complete integrability [1] [4], nice geometric formulations [8] [9] [10] and wave breaking phenomena [11] [12] [13] [14] etc. It was noticed that the peaked solitons were not allowed by the well-known in- 
tegrable equations such as the $\mathrm{KdV}$ equation, the $\mathrm{mKdV}$ equation, and the Schrodinger equation etc. The stability of peakons of the $\mathrm{CH}$ Equation (1.1) was proved in [15] [16]. A similar integrable equation with quadratic nonlinearities was the Degasperis-Procesi (DP) equation [17], which took the form

$$
m_{t}+3 u_{x} m+u m_{x}=0
$$

It was regarded as a model for nonlinear shallow water dynamics and could be derived from the governing equation for water waves [2]. Analogous to the $\mathrm{CH}$ equation, the DP equation also possessed an infinite number of conservation laws, bi-Hamiltonian structure, peaked solitons etc. Moreover, it admitted shocked peaked solitons [18]. Its integrability, existence of peaked solitons, stability of peaked solitons, and wave breaking phenomena were studied extensively in [19] [20] [21] [22].

Notice that both the $\mathrm{CH}$ and DP equations had the quadratic nonlinearities and $H^{1}$ conservation laws. So it was of great interest to search for such type of equations with higher-order nonlinearities. To the best of our knowledge, two integrable equations with cubic nonlinearities had been proposed. One was a modified $\mathrm{CH}$ equation

$$
m_{t}+\left(\left(u^{2}-u_{x}^{2}\right) m\right)_{x}=0
$$

which was obtained by the tri-Hamiltonian duality approach from the bi-Hamiltonian structure of the $\mathrm{mKdV}$ equation [5] [6]. Its well-posedness, blow-up, wave breaking, peaked solitons, and their stability were studied in recent works [23] [24] [25] [26]. The mCH Equation (1.3) exhibits new features of peaked solitons, wave-breaking mechanism, and blow-up criteria. The $\mathrm{mCH}$ equation could also be obtained from an invariant non-stretching planar curve flow in Euclidean geometry [24]. So it was regarded as an Euclidean version of the $\mathrm{CH}$ equation in this sense. The other one was the so-called Novikov equation

$$
m_{t}+3 u u_{x} m+u^{2} m_{x}=0
$$

which was obtained by Novikov [27] in the symmetry classification of such type equations. The integrability, existence of peaked solitons and their stability, global well-posedness and wave breaking phenomena of Equation (1.4) were discussed in [28] [29] [30] [31]. Applying the tri-Hamiltonian duality approach to the Gardner equation

$$
u_{t}+u_{x x x}+a_{1} u^{2} u_{x}+a_{2} u u_{x}=0
$$

they deduced the following generalized $\mathrm{CH}$ equation with the cubic and quadratic nonlinearities [32]

$$
m_{t}+a_{1}\left(\left(u^{2}-u_{x}^{2}\right) m\right)_{x}+a_{2}\left(2 u_{x} m+u m_{x}\right)=0
$$

This equation admitted Lax-pair and peaked solitons [33].

There are few studies on numerical solutions of the generalized Novikov equation. Therefore in this paper, we will construct a finite difference scheme for the initial boundary problem of equations as follows, 


$$
\begin{gathered}
m_{t}+a_{1}\left(3 u u_{x} m+u^{2} m_{x}\right)+a_{2} u_{x} m+a_{3} u m_{x}=0, \\
m=u-u_{x x}, 0<t \leq T, 0 \leq x \leq L \\
u(0, t)=u(L, t), 0<t \leq T \\
u(0, x)=u_{0}(x), 0<x \leq L
\end{gathered}
$$

where $u(x, t)$ is a function of time $t$ and a single spatial variable $x$, and $a_{1}$, $a_{2}, a_{3}$ are constants. It is clear that Equation (1.6) is reduced respectively to the Novikov Equation (1.4), the CH Equation (1.1) and the DP Equation (1.2), when $a_{1}=1, a_{2}=0, a_{3}=0 ; a_{1}=0, a_{2}=2, a_{3}=1 ; a_{1}=0, a_{2}=3, a_{3}=1$. Clearly, Equation (1.6) is a linear combination of the Novikov equation, the $\mathrm{CH}$ equation, and the DP equation.

We shall give an energy conservative finite scheme for Equations (1.6)-(1.8) and obtain the module estimation. Moreover, we prove the convergence and stability of the finite scheme by use of discrete energy method.

\section{Preliminaries}

For convenience, we denote $\Omega=\{(x, t) \mid 0 \leq x \leq L, 0<t \leq T\}$ in the following section. Let $N, J$ be any positive integers and $h=\frac{L}{J+1}, \tau=\frac{T}{N}, x_{j}=j h$ for $j=0,1, \cdots, J+1$. Denote $t^{n}=n \tau, u_{j}^{n}=u\left(x_{j}, t^{n}\right), u^{n}=\left(u_{0}^{n}, \cdots, u_{J+1}^{n}\right)$ for $n=0, \cdots, N$ and $Z_{h}^{0}=\left\{u=\left(u_{0}, \cdots, u_{J+1}\right) \mid u_{0}=u_{J+1}=0\right\}$.

For simplicity, we introduce some notations as follows:

$$
\begin{gathered}
u_{x}^{n}=\frac{u_{j+1}^{n}-u_{j}^{n}}{h}, u_{\bar{x}}^{n}=\frac{u_{j}^{n}-u_{j-1}^{n}}{h}, u_{\hat{x}}^{n}=\frac{u_{j+1}^{n}-u_{j-1}^{n}}{2 h}, \\
u_{t}^{n}=\frac{u_{j}^{n+1}-u_{j}^{n}}{\tau}, u_{\bar{t}}^{n}=\frac{u_{j}^{n}-u_{j}^{n-1}}{\tau}, u_{\hat{t}}^{n}=\frac{u_{j}^{n+1}-u_{j}^{n-1}}{2 \tau}, \\
\bar{u}=\frac{u_{j}^{n+1}+u_{j}^{n-1}}{2}, u^{n}=u_{j}^{n}
\end{gathered}
$$

For $u, v \in Z_{h}^{0}$, we define a discrete inner product and the discrete $L^{2}$-norm $\|\cdot\|_{h}$ as

$$
(u, v)_{h}=h \sum_{j=1}^{J} u_{j} v_{j},\|u\|_{h}=\left(h \sum_{j=1}^{J} u_{j}^{2}\right)^{\frac{1}{2}}
$$

In order to obtain the module estimation, investigate the convergence and stability of the finite difference scheme, we need introduce two lemmas as follows,

Lemma 2.1. (Discrete Sobolev inequality [34]) There exist constants $c_{1}, c_{2}$ satisfying

$$
\left\|u^{n}\right\|_{\infty} \leq c_{1}\left\|u^{n}\right\|+c_{2}\left\|u_{x}^{n}\right\|
$$

Lemma 2.2. (Discrete Gronwall inequality [34]) Suppose that there exist negative functions $\omega(k), \rho(k)$, where $\rho(k)$ is decreasing. For any $k$ and 
$c>0$, if

$$
\omega(k) \leq \rho(k)+c \tau \sum_{l=0}^{k-1} w(l)
$$

then

$$
\omega(k) \leq \rho(k) c^{c \tau k}
$$

\section{A Energy Conservative Finite Scheme}

The Equation (1.6) can be rewritten in the following form:

$$
u_{t}-u_{x x t}+a_{1}\left(3 u u_{x}\left(u-u_{x x}\right)+u^{2}\left(u_{x}-u_{x x x}\right)\right)+a_{2} u_{x}\left(u-u_{x x}\right)+a_{3} u\left(u_{x}-u_{x x x}\right)=0
$$

which can be expressed as

$$
u_{t}-u_{x x t}+4 a_{1} u^{2} u_{x}+\left(a_{2}+a_{3}\right) u u_{x}=3 a_{1} u u_{x} u_{x x}+a_{1} u^{2} u_{x x x}+a_{2} u_{x} u_{x x}+a_{3} u u_{x x x}
$$

Firstly, we construct an energy conservative finite scheme for the problem (1.6)-(1.8) as follows:

$$
\begin{gathered}
u_{t}^{n}-u_{t x \bar{x}}^{n}+a_{1}\left(\left(\left(u^{n+\frac{1}{2}}\right)^{3}\right)_{\bar{x}}+\left(u^{n+\frac{1}{2}}\right)^{2} u_{x}^{n+\frac{1}{2}}\right)+\frac{a_{2}+a_{3}}{3}\left(\left(\left(u^{n+\frac{1}{2}}\right)^{2}\right)_{\bar{x}}+u^{n+\frac{1}{2}} u_{x}^{n+\frac{1}{2}}\right) \\
=a_{1}\left(\left(\left(u^{n+\frac{1}{2}}\right)^{2} u_{x x}^{n+\frac{1}{2}}\right)_{\bar{x}}+u^{n+\frac{1}{2}} u_{x}^{n+\frac{1}{2}} u_{x x}^{n+\frac{1}{2}}\right)+a_{3}\left(\left(u^{n+\frac{1}{2}} u_{x x}^{n+\frac{1}{2}}\right)_{\bar{x}}+u_{x}^{n+\frac{1}{2}} u_{x x}^{n+\frac{1}{2}}\right) \\
+\left(a_{2}-2 a_{3}\right)\left(\left(\left(u_{x}^{n+\frac{1}{2}}\right)^{2}\right)_{\bar{x}}-u_{x}^{n+\frac{1}{2}} u_{x \bar{x}}^{n+\frac{1}{2}}\right) \\
u_{j}^{0}=u_{0}\left(x_{j}\right), \quad 0 \leq j \leq J+1
\end{gathered}
$$

and

$$
u_{0}^{n}=u_{J+1}^{n}=0, \quad 0 \leq n \leq N
$$

Lemma 3.1. The difference scheme satisfies discrete conservative law as follows:

$$
E^{n}=\left\|u^{n}\right\|^{2}+\left\|u_{x}^{n}\right\|^{2}=E^{n-1}=\cdots=E^{0}
$$

Proof. Computing the inner product of the difference scheme with $2 u^{n+\frac{1}{2}}$, we have

$$
\begin{aligned}
\left(u_{t}, 2 u^{n+\frac{1}{2}}\right) & =h \sum_{j=1}^{J} u_{t} \cdot\left(2 u^{n+\frac{1}{2}}\right)=\frac{h}{\tau} \sum_{j=1}^{J}\left(u_{j}^{n+1}-u_{j}^{n}\right)\left(u_{j}^{n+1}+u_{j}^{n}\right) \\
& =\frac{1}{\tau}\left(\left\|u^{n+1}\right\|^{2}-\left\|u^{n}\right\|^{2}\right) \\
\left(u_{t x \bar{x}}, 2 u^{n+\frac{1}{2}}\right) & =h \sum_{j=1}^{J} u_{t x \bar{x}} \cdot\left(2 u^{n+\frac{1}{2}}\right)=-\frac{h}{\tau} \sum_{j=1}^{J}\left(u_{j}^{n+1}-u_{j}^{n}\right)_{x}\left(u_{j}^{n+1}+u_{j}^{n}\right)_{x} \\
& =-\frac{1}{\tau}\left(\left\|u_{x}^{n+1}\right\|^{2}-\left\|u_{x}^{n}\right\|^{2}\right)
\end{aligned}
$$




$$
\begin{aligned}
& \left(a_{1}\left(\left(\left(u^{n+\frac{1}{2}}\right)^{3}\right)_{\bar{x}}+\left(u^{n+\frac{1}{2}}\right)^{2} u_{x}^{n+\frac{1}{2}}\right), 2 u^{n+\frac{1}{2}}\right) \\
& =2 a_{1} h \sum_{j=1}^{J}\left(\left(\left(u_{j}^{n+\frac{1}{2}}\right)^{3}\right)_{\bar{x}} \cdot u_{j}^{n+\frac{1}{2}}+\left(u_{j}^{n+\frac{1}{2}}\right)^{2} u_{x j}^{n+\frac{1}{2}} \cdot u_{j}^{n+\frac{1}{2}}\right) \\
& =2 a_{1} h \sum_{j=1}^{J}\left(\left(\left(u_{j}^{n+\frac{1}{2}}\right)^{3}\right)_{\bar{x}} \cdot u_{j}^{n+\frac{1}{2}}-\left(\left(u_{j}^{n+\frac{1}{2}}\right)^{3}\right)_{\bar{x}} u_{j}^{n+\frac{1}{2}}\right) \\
& =0 \\
& \left(\frac{a_{2}+a_{3}}{3}\left(\left(\left(u^{n+\frac{1}{2}}\right)^{2}\right)_{\bar{x}}+u^{n+\frac{1}{2}} u_{x}^{n+\frac{1}{2}}\right), 2 u^{n+\frac{1}{2}}\right) \\
& =\frac{2\left(a_{2}+a_{3}\right)}{3} h \sum_{j=1}^{J}\left(\left(\left(u_{j}^{n+\frac{1}{2}}\right)^{2}\right)_{\bar{x}} \cdot u_{j}^{n+\frac{1}{2}}+u_{j}^{n+\frac{1}{2}} u_{x j}^{n+\frac{1}{2}} \cdot u_{j}^{n+\frac{1}{2}}\right) \\
& =\frac{2\left(a_{2}+a_{3}\right)}{3} h \sum_{j=1}^{J}\left(\left(\left(u_{j}^{n+\frac{1}{2}}\right)^{2}\right)_{\bar{x}} \cdot u_{j}^{n+\frac{1}{2}}-\left(\left(u_{j}^{n+\frac{1}{2}}\right)^{2}\right)_{\bar{x}} u_{j}^{n+\frac{1}{2}}\right) \\
& =0 \\
& \left(a_{1}\left(\left(\left(u^{n+\frac{1}{2}}\right)^{2} u_{x x}^{n+\frac{1}{2}}\right)_{\bar{x}}+u^{n+\frac{1}{2}} u_{x}^{n+\frac{1}{2}} u_{x x}^{n+\frac{1}{2}}\right), 2 u^{n+\frac{1}{2}}\right) \\
& =2 a_{1} h \sum_{j=1}^{J}\left(\left(\left(u_{j}^{n+\frac{1}{2}}\right)^{2} u_{x x j}^{n+\frac{1}{2}}\right)_{\bar{x}} \cdot u_{j}^{n+\frac{1}{2}}+u_{j}^{n+\frac{1}{2}} u_{x j}^{n+\frac{1}{2}} u_{x x j}^{n+\frac{1}{2}} \cdot u_{j}^{n+\frac{1}{2}}\right) \\
& =2 a_{1} h \sum_{j=1}^{J}\left(\left(\left(u_{j}^{n+\frac{1}{2}}\right)^{2} u_{x x j}^{n+\frac{1}{2}}\right)_{\bar{x}} \cdot u_{j}^{n+\frac{1}{2}}-\left(\left(u_{j}^{n+\frac{1}{2}}\right)^{2} u_{x x j}^{n+\frac{1}{2}}\right)_{\bar{x}} u_{j}^{n+\frac{1}{2}}\right) \\
& =0 \\
& \left(a_{3}\left(\left(u^{n+\frac{1}{2}} u_{x x}^{n+\frac{1}{2}}\right)_{\bar{x}}+u_{x}^{n+\frac{1}{2}} u_{x x}^{n+\frac{1}{2}}\right), 2 u^{n+\frac{1}{2}}\right) \\
& =2 a_{3} h \sum_{j=1}^{J}\left(\left(u_{j}^{n+\frac{1}{2}} u_{x x j}^{n+\frac{1}{2}}\right)_{\bar{x}} \cdot u_{j}^{n+\frac{1}{2}}+u_{x j}^{n+\frac{1}{2}} u_{x x j}^{n+\frac{1}{2}} \cdot u_{j}^{n+\frac{1}{2}}\right) \\
& =2 a_{3} h \sum_{j=1}^{J}\left(\left(u_{j}^{n+\frac{1}{2}} u_{x x j}^{n+\frac{1}{2}}\right)_{\bar{x}} \cdot u_{j}^{n+\frac{1}{2}}-u_{j}^{n+\frac{1}{2}}\left(u_{j}^{n+\frac{1}{2}} u_{x x j}^{n+\frac{1}{2}}\right)_{\bar{x}}\right) \\
& =0 \\
& \left(\left(a_{2}-2 a_{3}\right)\left(\left(\left(u_{x}^{n+\frac{1}{2}}\right)^{2}\right)_{\bar{x}}-u_{x}^{n+\frac{1}{2}} u_{x \bar{x}}^{n+\frac{1}{2}}\right), 2 u^{n+\frac{1}{2}}\right) \\
& =2\left(a_{2}-2 a_{3}\right) h \sum_{j=1}^{J}\left(\left(\left(u_{x j}^{n+\frac{1}{2}}\right)^{2}\right)_{\bar{x}} \cdot u_{j}^{n+\frac{1}{2}}-u_{x j}^{n+\frac{1}{2}} u_{x \overline{x j}}^{n+\frac{1}{2}} \cdot u_{j}^{n+\frac{1}{2}}\right) \\
& =2\left(a_{2}-2 a_{3}\right) h \sum_{j=1}^{J}\left(-\left(u_{x j}^{n+\frac{1}{2}}\right)^{2} \cdot u_{x j}^{n+\frac{1}{2}}+u_{x j}^{n+\frac{1}{2}} u_{x j}^{n+\frac{1}{2}} \cdot u_{x j}^{n+\frac{1}{2}}\right)
\end{aligned}
$$


From the above formulas, we can simplify formula as

$$
\frac{1}{\tau}\left(\left\|u^{n+1}\right\|^{2}-\left\|u^{n}\right\|^{2}\right)+\frac{1}{\tau}\left(\left\|u_{x}^{n+1}\right\|^{2}-\left\|u_{x}^{n}\right\|^{2}\right)=0
$$

then

$$
\left\|u^{n+1}\right\|^{2}+\left\|u_{x}^{n+1}\right\|^{2}=\left\|u^{n}\right\|^{2}+\left\|u_{x}^{n}\right\|^{2}
$$

So the difference scheme satisfies the discrete conservative law as follows

$$
E^{n}=\left\|u^{n}\right\|^{2}+\left\|u_{x}^{n}\right\|^{2}=E^{n-1}=\cdots=E^{0}
$$

This completes the proof of Lemma 3.1.

Theorem 3.2. The solution of the difference scheme (3.1)-(3.3) satisfies:

$$
\left\|u^{n}\right\| \leq C, \quad\left\|u_{x}^{n}\right\| \leq C,\left\|u^{n}\right\|_{\infty} \leq C
$$

Proof. From Lemma 3.1, we know $\left\|u^{n}\right\| \leq C$ and $\left\|u_{x}^{n}\right\| \leq C$. Then by Lemma 2.1, we obtain $\left\|u^{n}\right\|_{\infty} \leq C$. This completes the proof of Theorem 3.2.

Theorem 3.3. There exists $u^{n} \in Z_{h}^{0}$ satisfying the difference scheme (3.1)-(3.3).

Proof. We shall use Mathematical Induction to prove Theorem 3.3.

When $n=1$, it is known that there exists $u^{1}$ satisfying the difference scheme from initial condition. Next, we need to prove the case of $n>1$.

Assume that there exists $u^{n}$ satisfies the difference scheme when $n<N$, then we need to prove that there exists $u^{n+1}$ satisfies the difference scheme. Define a operator $\omega(v)$ in $Z_{h}^{0}$ as follows:

$$
\begin{aligned}
\omega(v)= & 2 v-2 u^{n}-\left(2 v_{x \bar{x}}-2 u_{x \bar{x}}^{n}\right)+a_{1} \tau\left(\left(v^{3}\right)_{\bar{x}}+v^{2} v_{x}\right) \\
& +\frac{a_{2}+a_{3}}{3} \tau\left(\left(v^{2}\right)_{\bar{x}}+v v_{x}\right)-a_{1} \tau\left(\left(v^{2} v_{x x}\right)_{\bar{x}}+v v_{x} v_{x x}\right) \\
& -a_{3} \tau\left(\left(v v_{x x}\right)_{\bar{x}}+v_{x} v_{x x}\right)-\left(a_{2}-2 a_{3}\right) \tau\left(\left(\left(v_{x}\right)^{2}\right)_{\bar{x}}-v_{x} v_{x \bar{x}}\right)
\end{aligned}
$$

It is clear that $\omega$ is continuous.

Computing the inner product of the operator $\omega(v)$, we obtain

$$
\begin{gathered}
(2 v, v)=2\|v\|^{2} \\
\left(2 v_{x \bar{x}}, v\right)=-2\left(v_{x}, v_{x}\right)=-2\left\|v_{x}\right\|^{2} \\
\left(\left(v^{3}\right)_{\bar{x}}+v^{2} v_{x}, v\right)=h \sum_{j=1}^{J}\left(\left(v^{3}\right)_{\bar{x}} \cdot v+v^{2} v_{x} \cdot v\right)=0 \\
\left(\left(v^{2}\right)_{\bar{x}}+v v_{x}, v\right)=h \sum_{j=1}^{J}\left(\left(v^{2}\right)_{\bar{x}} \cdot v+v v_{x} \cdot v\right)=0 \\
\left(\left(v^{2} v_{x x}\right)_{\bar{x}}+v v_{x} v_{x x}, v\right)=h \sum_{j=1}^{J}\left(\left(v^{2} v_{x x}\right)_{\bar{x}} \cdot v+v v_{x} v_{x x} \cdot v\right)=0 \\
\left(\left(v v_{x x}\right)_{\bar{x}}+v_{x} v_{x x}, v\right)=h \sum_{j=1}^{J}\left(\left(v v_{x x}\right)_{\bar{x}} \cdot v+v_{x} v_{x x} \cdot v\right)=0
\end{gathered}
$$




$$
\left(\left(\left(v_{x}\right)^{2}\right)_{\bar{x}}-v_{x} v_{x \bar{x}}, v\right)=h \sum_{j=1}^{J}\left(-\left(v_{x}\right)^{2} \cdot v_{x}+v_{x} v_{x} \cdot v_{x}\right)=0
$$

By using Cauchy-Schwartz inequality, we obtain

$$
\begin{gathered}
\left(2 u^{n}, v\right) \leq 2\left\|u^{n}\right\| \cdot\|v\| \\
\left(2 u_{x \bar{x}}^{n}, v\right)=-2\left(u_{x}^{n}, v_{x}\right) \geq-2\left\|u_{x}^{n}\right\| \cdot\left\|v_{x}\right\|
\end{gathered}
$$

From above discussions, we get

$$
\begin{aligned}
(\omega(v), v) & \geq 2\|v\|^{2}-2\left\|u^{n}\right\| \cdot\|v\|+2\left\|v_{x}\right\|^{2}-2\left\|u_{x}^{n}\right\| \cdot\left\|v_{x}\right\| \\
& \geq\|v\|^{2}-\left\|u^{n}\right\|^{2}-\left\|u_{x}^{n}\right\|^{2}
\end{aligned}
$$

For $\forall v \in Z_{h}^{0}$, then

$$
\|v\|^{2}=\left\|u^{n}\right\|^{2}+\left\|u_{x}^{n}\right\|^{2}+1
$$

So we get $(\omega(v), v)>0$. By Brouwer fixed point theorem, there exists $v^{*} \in H$ such that $\omega\left(v^{*}\right)=0$ and $\left\|v^{*}\right\| \leq \partial$.

Let $u^{n+1}=2 v^{*}-u^{n}$, it is easy to verify that $u^{n+1}$ satisfies the difference scheme for the problem.

So we complete the proof of Theorem 3.3.

\section{Convergence and Stability of the Difference Scheme}

In order to investigate the convergence and stability of the difference scheme, we need to obtain the truncation error of the scheme.

Theorem 4.1. If the solution $u(x, t)$ of equation is sufficiently regular, then the truncation error of the difference scheme is $R_{j}^{n}=O\left(\tau^{2}+h^{2}\right)$.

Proof. Firstly, we can use the Taylor expansion of $u_{j}^{n+1}, u_{j}^{n}, u_{j+1}^{n+1}, u_{j+1}^{n}$, $u_{j-1}^{n+1}, u_{j-1}^{n}$ at the point $\left(x_{j}, t_{n+\frac{1}{2}}\right)$. Secondly, from the above Taylor expansions we reorganize difference equation at point $\left(x_{j}, t_{n+\frac{1}{2}}\right)$, then

$$
\begin{aligned}
\left(x_{j}, t_{n+\frac{1}{2}}\right) u_{t}^{n}= & \frac{u_{j}^{n+1}-u_{j}^{n}}{\tau} \\
= & \frac{1}{\tau}\left(\left(u_{j}^{n+\frac{1}{2}}+\frac{\tau}{2}\left(\frac{\partial u}{\partial t}\right)_{j}^{n+\frac{1}{2}}+\frac{\tau^{2}}{8}\left(\frac{\left.\partial^{2} u\right)^{n+\frac{1}{2}}}{\partial t^{2}}\right)_{j}+\frac{\tau^{3}}{48}\left(\frac{\partial^{3} u}{\partial t^{3}}\right)_{j}^{n+\frac{1}{2}}+\cdots\right)\right. \\
& \left.-\left(u_{j}^{n+\frac{1}{2}}-\frac{\tau}{2}\left(\frac{\partial u}{\partial t}\right)_{j}^{n+\frac{1}{2}}+\frac{\tau^{2}}{8}\left(\frac{\partial^{2} u}{\partial t^{2}}\right)_{j}^{n+\frac{1}{2}}+\cdots\right)\right) \\
= & \left(\frac{\partial u}{\partial t}\right)_{j}^{n+\frac{1}{2}}+\frac{\tau^{3}}{24}\left(\frac{\partial^{3} u}{\partial t^{3}}\right)_{j}^{n+\frac{1}{2}}+\cdots
\end{aligned}
$$




$$
\begin{aligned}
& u_{t \bar{x}}^{n}=\frac{\left(u_{x \bar{x}}\right)_{j}^{n+1}-\left(u_{x \bar{x}}\right)_{j}^{n}}{\tau}=\frac{\left(\left(u_{\bar{x}}\right)_{j+1}^{n+1}-\left(u_{\bar{x}}\right)_{j}^{n+1}\right)-\left(\left(u_{\bar{x}}\right)_{j+1}^{n}-\left(u_{\bar{x}}\right)_{j}^{n}\right)}{\tau h} \\
& =\frac{1}{h^{2}}\left(\left(\left(\frac{\partial u}{\partial t}\right)_{j+1}^{n+\frac{1}{2}}-2\left(\frac{\partial u}{\partial t}\right)_{j}^{n+\frac{1}{2}}+\left(\frac{\partial u}{\partial t}\right)_{j-1}^{n+\frac{1}{2}}\right)\right. \\
& \left.+\left(\frac{\tau^{2}}{24}\left(\frac{\partial^{3} u}{\partial t^{3}}\right)_{j+1}^{n+\frac{1}{2}}-\frac{\tau^{2}}{12}\left(\frac{\partial^{3} u}{\partial t^{3}}\right)_{j}^{n+\frac{1}{2}}+\frac{\tau^{2}}{24}\left(\frac{\partial^{3} u}{\partial t^{3}}\right)_{j-1}^{n+\frac{1}{2}}\right)+\cdots\right) \\
& =\left(\frac{\partial^{3} u}{\partial t \partial x^{2}}\right)_{j}^{n+\frac{1}{2}}+\cdots \\
& \left(\left(u^{n+\frac{1}{2}}\right)^{3}\right)_{\bar{x}}=\frac{\left(\left(u^{n+\frac{1}{2}}\right)^{3}\right)_{j}-\left(\left(u^{n+\frac{1}{2}}\right)^{3}\right)_{j-1}}{h} \\
& =3\left(u^{n+\frac{1}{2}}\right)^{2}\left(\frac{\partial u}{\partial x}\right)_{j}^{n+\frac{1}{2}}-\frac{h}{2}\left(3\left(u^{n+\frac{1}{2}}\right)^{2}\left(\frac{\partial^{2} u}{\partial x^{2}}\right)_{j}^{n+\frac{1}{2}}+6 u^{n+\frac{1}{2}}\left(\frac{\partial u}{\partial x}\right)_{j}^{n+\frac{1}{2}}+\cdots\right) \\
& \left(u^{n+\frac{1}{2}}\right)^{2} u_{x}^{n+\frac{1}{2}}=\left(u^{n+\frac{1}{2}}\right)^{2} \cdot \frac{u_{j+1}^{n+\frac{1}{2}}-u_{j}^{n+\frac{1}{2}}}{h} \\
& =\left(u^{n+\frac{1}{2}}\right)^{2} \cdot\left(\left(\frac{\partial u}{\partial x}\right)_{j}^{n+\frac{1}{2}}+\frac{h}{2}\left(\frac{\partial^{2} u}{\partial x^{2}}\right)_{j}^{n+\frac{1}{2}}+\frac{h^{2}}{6}\left(\frac{\partial^{3} u}{\partial x^{3}}\right)_{j}^{n+\frac{1}{2}}+\cdots\right) \\
& \left(\left(u^{n+\frac{1}{2}}\right)^{2}\right)_{\bar{x}}=\frac{\left(\left(u^{n+\frac{1}{2}}\right)^{2}\right)_{j}-\left(\left(u^{n+\frac{1}{2}}\right)^{2}\right)_{j-1}}{h} \\
& =2 u^{n+\frac{1}{2}}\left(\frac{\partial u}{\partial x}\right)_{j}^{n+\frac{1}{2}}-\frac{h}{2}\left(2 u^{n+\frac{1}{2}}\left(\frac{\partial^{2} u}{\partial x^{2}}\right)_{j}^{n+\frac{1}{2}}+2\left(\frac{\partial u}{\partial x}\right)_{j}^{n+\frac{1}{2}}+\cdots\right) \\
& u^{n+\frac{1}{2}} u_{x}^{n+\frac{1}{2}}=u^{n+\frac{1}{2}} \cdot \frac{u_{j+1}^{n+\frac{1}{2}}-u_{j}^{n+\frac{1}{2}}}{h} \\
& =u^{n+\frac{1}{2}} \cdot\left(\left(\frac{\partial u}{\partial x}\right)_{j}^{n+\frac{1}{2}}+\frac{h}{2}\left(\frac{\partial^{2} u}{\partial x^{2}}\right)_{j}^{n+\frac{1}{2}}+\frac{h^{2}}{6}\left(\frac{\partial^{3} u}{\partial x^{3}}\right)_{j}^{n+\frac{1}{2}}+\cdots\right) \\
& u^{n+\frac{1}{2}} u_{x}^{n+\frac{1}{2}} u_{x x}^{n+\frac{1}{2}}=u^{n+\frac{1}{2}} \cdot \frac{u_{j+1}^{n+\frac{1}{2}}-u_{j}^{n+\frac{1}{2}}}{h} \cdot \frac{u_{j+2}^{n+\frac{1}{2}}-2 u_{j+1}^{n+\frac{1}{2}}+u_{j}^{n+\frac{1}{2}}}{h^{2}} \\
& =u^{n+\frac{1}{2}} \cdot\left(\left(\frac{\partial u}{\partial x}\right)_{j}^{n+\frac{1}{2}}+\frac{h}{2}\left(\frac{\partial^{2} u}{\partial x^{2}}\right)_{j}^{n+\frac{1}{2}}+\cdots\right) \cdot\left(\left(\frac{\partial^{2} u}{\partial x^{2}}\right)_{j}^{n+\frac{1}{2}}+h\left(\frac{\partial^{3} u}{\partial x^{3}}\right)_{j}^{n+\frac{1}{2}}+\cdots\right)
\end{aligned}
$$




$$
\begin{aligned}
& \left(\left(u^{n+\frac{1}{2}}\right)^{2} u_{x x}^{n+\frac{1}{2}}\right)_{\bar{x}}=\frac{\left(u_{j}^{n+\frac{1}{2}}\right)^{2} u_{x x j}^{n+\frac{1}{2}}-\left(u_{j-1}^{n+\frac{1}{2}}\right)^{2} u_{x x(j-1)}^{n+\frac{1}{2}}}{h} \\
& =2 u^{n+\frac{1}{2}} \cdot\left(\left(\frac{\partial u}{\partial x}\right)_{j}^{n+\frac{1}{2}} \cdot\left(\frac{\partial^{2} u}{\partial x^{2}}\right)_{j}^{n+\frac{1}{2}}+\left(\frac{\partial^{3} u}{\partial x^{3}}\right)_{j}^{n+\frac{1}{2}}+h\left(\frac{\partial^{3} u}{\partial x^{3}}\right)_{j}^{n+\frac{1}{2}}+\cdots\right) \\
& u_{x}^{x+\frac{1}{2}} u_{x x}^{x+\frac{1}{2}}=\frac{u_{j+1}^{x+\frac{1}{2}}-u_{j}^{x+\frac{1}{2}}}{h} \cdot \frac{u_{j+2}^{x+\frac{1}{2}}-2 u_{j+1}^{x+\frac{1}{2}}+u_{j}^{x+\frac{1}{2}}}{h^{2}} \\
& =\left(\left(\frac{\partial u}{\partial x}\right)_{j}^{n+\frac{1}{2}}+\frac{h}{2}\left(\frac{\partial^{2} u}{\partial x^{2}}\right)_{j}^{n+\frac{1}{2}}+\cdots\right) \cdot\left(\left(\frac{\partial^{2} u}{\partial x^{2}}\right)_{j}^{n+\frac{1}{2}}+h\left(\frac{\partial^{3} u}{\partial x^{3}}\right)_{j}^{n+\frac{1}{2}}+\cdots\right) \\
& \left(u^{n+\frac{1}{2}} u_{x x}^{n+\frac{1}{2}}\right)_{\bar{x}}=\frac{u_{j}^{n+\frac{1}{2}} u_{x x j}^{n+\frac{1}{2}}-u_{j-1}^{n+\frac{1}{2}} u_{x x(j-1)}^{n+\frac{1}{2}}}{h} \\
& =\left(\frac{\partial u}{\partial x}\right)_{j}^{n+\frac{1}{2}} \cdot\left(\frac{\partial^{2} u}{\partial x^{2}}\right)_{j}^{n+\frac{1}{2}}+\left(\frac{\partial^{3} u}{\partial x^{3}}\right)_{j}^{n+\frac{1}{2}}+h\left(\frac{\partial^{3} u}{\partial x^{3}}\right)_{j}^{n+\frac{1}{2}}+\cdots \\
& \left(\left(u_{x}^{n+\frac{1}{2}}\right)^{2}\right)_{\bar{x}}=\frac{u_{x j}^{n+\frac{1}{2}} u_{x j}^{n+\frac{1}{2}}-u_{x(j-1)}^{n+\frac{1}{2}} u_{x(j-1)}^{n+\frac{1}{2}}}{h} \\
& =\left(\frac{\partial u}{\partial x}\right)_{j}^{n+\frac{1}{2}} \cdot\left(\frac{\partial^{2} u}{\partial x^{2}}\right)_{j}^{n+\frac{1}{2}}+\left(\frac{\partial^{3} u}{\partial x^{3}}\right)_{j}^{n+\frac{1}{2}}+h\left(\frac{\partial^{3} u}{\partial x^{3}}\right)_{j}^{n+\frac{1}{2}}+\cdots \\
& u_{x}^{n+\frac{1}{2}} u_{x \bar{x}}^{n+\frac{1}{2}}=\frac{u_{j+1}^{n+\frac{1}{2}}-u_{j}^{n+\frac{1}{2}}}{h} \cdot \frac{u_{j+1}^{n+\frac{1}{2}}-2 u_{j}^{n+\frac{1}{2}}+u_{j-1}^{n+\frac{1}{2}}}{h^{2}} \\
& =\left(\left(\frac{\partial u}{\partial x}\right)_{j}^{n+\frac{1}{2}}+\frac{h}{2}\left(\frac{\partial^{2} u}{\partial x^{2}}\right)_{j}^{n+\frac{1}{2}}+\cdots\right) \cdot\left(\left(\frac{\partial^{2} u}{\partial x^{2}}\right)_{j}^{n+\frac{1}{2}}+h\left(\frac{\partial^{3} u}{\partial x^{3}}\right)_{j}^{n+\frac{1}{2}}+\cdots\right)
\end{aligned}
$$

From the above expansions, we obtain the linear part of Equation (3.1) $u_{t}^{n}-u_{t x \bar{x}}^{n}$ at the point $\left(x_{j}, t_{n+\frac{1}{2}}\right)$ satisfying

$$
u_{t}^{n}-u_{t x \bar{x}}^{n}=\left(\frac{\partial u}{\partial t}-\frac{\partial^{3} u}{\partial t \partial x^{2}}\right){ }_{\left.x_{j, t},{ }_{n+\frac{1}{2}}\right)}+O\left(\tau^{2}+h^{2}\right)
$$

On the other hand, the nonlinear parts at the point $\left(x_{j}, t_{n+\frac{1}{2}}\right)$ satisfying

$$
\begin{gathered}
a_{1}\left(\left(\left(u^{n+\frac{1}{2}}\right)^{3}\right)_{\bar{x}}+\left(u^{n+\frac{1}{2}}\right)^{2} u_{x}^{n+\frac{1}{2}}\right)=4 a_{1} u^{2}\left(\frac{\partial u}{\partial x}\right)_{j}^{n+\frac{1}{2}}+O\left(\tau^{2}+h^{2}\right) \\
\frac{a_{2}+a_{3}}{3}\left(\left(\left(u^{n+\frac{1}{2}}\right)^{2}\right)+u^{n+\frac{1}{2}} u_{x}^{n+\frac{1}{2}}\right)=\left(a_{2}+a_{3}\right) u\left(\frac{\partial u}{\partial x}\right)_{j}^{n+\frac{1}{2}}+O\left(\tau^{2}+h^{2}\right)
\end{gathered}
$$




$$
\begin{aligned}
& a_{1}\left(\left(\left(u^{n+\frac{1}{2}}\right)^{2} u_{x x}^{n+\frac{1}{2}}\right)_{\bar{x}}+u^{n+\frac{1}{2}} u_{x}^{n+\frac{1}{2}} u_{x x}^{n+\frac{1}{2}}\right) \\
& =a_{1}\left(3 u\left(\frac{\partial u}{\partial x} \cdot \frac{\partial^{2} u}{\partial x^{2}}\right)_{j}^{n+\frac{1}{2}}+2 u\left(\frac{\partial^{3} u}{\partial x^{3}}\right)_{j}^{n+\frac{1}{2}}\right)+O\left(\tau^{2}+h^{2}\right) \\
& a_{3}\left(\left(u^{n+\frac{1}{2}} u_{x x}^{n+\frac{1}{2}}\right)_{\bar{x}}+u_{x}^{n+\frac{1}{2}} u_{x x}^{n+\frac{1}{2}}\right)=a_{3}\left(2\left(\frac{\partial u}{\partial x} \cdot \frac{\partial^{2} u}{\partial x^{2}}\right)_{j}^{n+\frac{1}{2}}+\left(\frac{\partial^{3} u}{\partial x^{3}}\right)_{j}^{n+\frac{1}{2}}\right)+O\left(\tau^{2}+h^{2}\right) \\
& \left(a_{2}-2 a_{3}\right)\left(\left(\left(u_{x}^{n+\frac{1}{2}}\right)^{2}\right)_{\bar{x}}-u_{x}^{n+\frac{1}{2}} u_{x \bar{x}}^{n+\frac{1}{2}}\right) \\
& =\left(a_{2}-2 a_{3}\right)\left(2\left(\frac{\partial u}{\partial x} \cdot \frac{\partial^{2} u}{\partial x^{2}}\right)_{j}^{n+\frac{1}{2}}+\left(\frac{\partial^{3} u}{\partial x^{3}}\right)_{j}^{n+\frac{1}{2}}\right)+O\left(\tau^{2}+h^{2}\right)
\end{aligned}
$$

Then we can easily obtain that truncation error of scheme (3.1) is $R_{j}^{n}=O\left(\tau^{2}+h^{2}\right)$.

Theorem 4.2. The solution of the difference scheme (3.1)-(3.3) approaches to the solution of the original differential Equations (1.6)-(1.8) in $L_{\infty}$, and the corresponding truncation error is $O\left(\tau^{2}+h^{2}\right)$.

Proof. Assume that $u_{j}^{n}$ is the solution of the difference scheme (3.1)-(3.3), $U_{j}^{n}$ is the solution of the original differential Equations (1.6)-(1.8). We can easily have $e_{j}^{n}=U_{j}^{n}-u_{j}^{n}$.

For the difference scheme (3.1),

$$
\begin{aligned}
R^{n}= & U_{t}^{n}-U_{t x \bar{x}}^{n}+a_{1}\left(\left(\left(U^{n+\frac{1}{2}}\right)^{3}\right)_{\bar{x}}+\left(U^{n+\frac{1}{2}}\right)^{2} U_{x}^{n+\frac{1}{2}}\right) \\
& +\frac{a_{2}+a_{3}}{3}\left(\left(\left(U^{n+\frac{1}{2}}\right)^{2}\right)_{\bar{x}}+U^{n+\frac{1}{2}} U_{x}^{n+\frac{1}{2}}\right)-a_{1}\left(\left(\left(U^{n+\frac{1}{2}}\right)^{2} U_{x x}^{n+\frac{1}{2}}\right)_{\bar{x}}+U^{n+\frac{1}{2}} U_{x}^{n+\frac{1}{2}} U_{x x}^{n+\frac{1}{2}}\right) \\
& -a_{3}\left(\left(U^{n+\frac{1}{2}} U_{x x}^{n+\frac{1}{2}}\right)_{\bar{x}}+U_{x}^{n+\frac{1}{2}} U_{x x}^{n+\frac{1}{2}}\right)-\left(a_{2}-2 a_{3}\right)\left(\left(U_{x}^{n+\frac{1}{2}}\right)^{2}\right) U_{\bar{x}}^{n+\frac{1}{2}} U_{x \bar{x}}^{n+\frac{1}{2}}
\end{aligned}
$$

then $(4,1)$ minus (3.1), yields

$$
R^{n}=e_{t}^{n}-e_{t x \bar{x}}^{n}+I_{1}+I_{2}+I_{3}+I_{4}+I_{5}
$$

where

$$
\begin{gathered}
I_{1}=a_{1}\left(\left(\left(U^{n+\frac{1}{2}}\right)^{3}\right)_{\bar{x}}+\left(U^{n+\frac{1}{2}}\right)^{2} U_{x}^{n+\frac{1}{2}}-\left(\left(u^{n+\frac{1}{2}}\right)^{3}\right)_{\bar{x}}-\left(u^{n+\frac{1}{2}}\right)^{2} u_{x}^{n+\frac{1}{2}}\right) \\
I_{2}=\frac{a_{2}+a_{3}}{3}\left(\left(\left(U^{n+\frac{1}{2}}\right)^{2}\right)_{\bar{x}}+U^{n+\frac{1}{2}} U_{x}^{n+\frac{1}{2}}-\left(\left(u^{n+\frac{1}{2}}\right)^{2}\right)_{\bar{x}}-u^{n+\frac{1}{2}} u_{x}^{n+\frac{1}{2}}\right)
\end{gathered}
$$




$$
\begin{gathered}
I_{3}=a_{1}\left(\left(\left(u^{n+\frac{1}{2}}\right)^{2} u_{x x}^{n+\frac{1}{2}}\right)_{\bar{x}}+u^{n+\frac{1}{2}} u_{x}^{n+\frac{1}{2}} u_{x x}^{n+\frac{1}{2}}-\left(\left(U^{n+\frac{1}{2}}\right)^{2} U_{x x}^{n+\frac{1}{2}}\right)_{\bar{x}}-U^{n+\frac{1}{2}} U_{x}^{n+\frac{1}{2}} U_{x x}^{n+\frac{1}{2}}\right) \\
I_{4}=a_{3}\left(\left(u^{n+\frac{1}{2}} u_{x x}^{n+\frac{1}{2}}\right)_{\bar{x}}+u_{x}^{n+\frac{1}{2}} u_{x x}^{n+\frac{1}{2}}-\left(U^{n+\frac{1}{2}} U_{x x}^{n+\frac{1}{2}}\right)_{\bar{x}}-U_{x}^{n+\frac{1}{2}} U_{x x}^{n+\frac{1}{2}}\right) \\
I_{5}=\left(a_{2}-2 a_{3}\right)\left(\left(\left(u_{x}^{n+\frac{1}{2}}\right)^{2}\right)-u_{x}^{n+\frac{1}{2}} u_{x \bar{x}}^{n+\frac{1}{2}}-\left(\left(U_{x}^{n+\frac{1}{2}}\right)^{2}\right)_{\bar{x}}+U_{x}^{n+\frac{1}{2}} U_{x \bar{x}}^{n+\frac{1}{2}}\right)
\end{gathered}
$$

Taking the inner product of (4.2) with $2 e^{n+\frac{1}{2}}$, we obtain

$$
\begin{aligned}
\left(R^{n}, 2 e^{n+\frac{1}{2}}\right)= & \left(e_{t}^{n}, 2 e^{n+\frac{1}{2}}\right)-\left(e_{t x \bar{x}}^{n}, 2 e^{n+\frac{1}{2}}\right)+\left(I_{1}, 2 e^{n+\frac{1}{2}}\right)+\left(I_{2}, 2 e^{n+\frac{1}{2}}\right) \\
& +\left(I_{3}, 2 e^{n+\frac{1}{2}}\right)+\left(I_{4}, 2 e^{n+\frac{1}{2}}\right)+\left(I_{5}, 2 e^{n+\frac{1}{2}}\right)
\end{aligned}
$$

By computing $\left(e_{t}^{n}, 2 e^{n+\frac{1}{2}}\right),\left(e_{t \bar{x}}^{n}, 2 e^{n+\frac{1}{2}}\right),\left(I_{1}, 2 e^{n+\frac{1}{2}}\right),\left(I_{2}, 2 e^{n+\frac{1}{2}}\right)$, $\left(I_{3}, 2 e^{n+\frac{1}{2}}\right),\left(I_{4}, 2 e^{n+\frac{1}{2}}\right)$ and $\left(I_{5}, 2 e^{n+\frac{1}{2}}\right)$ respectively, and from Lemma 2.3 and Cauchy-Schwartz inequality, we have

$$
\begin{aligned}
& \left(e_{t}^{n}, 2 e^{n+\frac{1}{2}}\right)=h \sum_{j=0}^{J} e_{t j} \cdot 2 e_{j}^{n+\frac{1}{2}}=\frac{1}{\tau}\left(\left\|e^{n+1}\right\|^{2}-\left\|e^{n}\right\|^{2}\right) \\
& \left(e_{t x \bar{x}}^{n}, 2 e^{n+\frac{1}{2}}\right)=h \sum_{j=0}^{J} e_{t x \bar{x} j} \cdot 2 e_{j}^{n+\frac{1}{2}}=\frac{1}{\tau}\left(\left\|e_{x}^{n+1}\right\|^{2}-\left\|e_{x}^{n}\right\|^{2}\right) \\
& \left(I_{1}, 2 e^{n+\frac{1}{2}}\right) \leq C\left(\left\|e_{x}^{n+1}\right\|^{2}+\left\|e_{x}^{n}\right\|^{2}+\left\|e^{n+1}\right\|^{2}+\left\|e^{n}\right\|^{2}\right) \\
& \left(I_{2}, 2 e^{n+\frac{1}{2}}\right) \leq C\left(\left\|e_{x}^{n+1}\right\|^{2}+\left\|e_{x}^{n}\right\|^{2}+\left\|e^{n+1}\right\|^{2}+\left\|e^{n}\right\|^{2}\right) \\
& \left(I_{3}, 2 e^{n+\frac{1}{2}}\right) \leq C\left(\left\|e_{x}^{n+1}\right\|^{2}+\left\|e_{x}^{n}\right\|^{2}+\left\|e^{n+1}\right\|^{2}+\left\|e^{n}\right\|^{2}\right) \\
& \left(I_{4}, 2 e^{n+\frac{1}{2}}\right) \leq C\left(\left\|e_{x}^{n+1}\right\|^{2}+\left\|e_{x}^{n}\right\|^{2}+\left\|e^{n+1}\right\|^{2}+\left\|e^{n}\right\|^{2}\right) \\
& \left(I_{5}, 2 e^{n+\frac{1}{2}}\right) \leq C\left(\left\|e_{x}^{n+1}\right\|^{2}+\left\|e_{x}^{n}\right\|^{2}+\left\|e^{n+1}\right\|^{2}+\left\|e^{n}\right\|^{2}\right)
\end{aligned}
$$

Therefore, 


$$
\begin{aligned}
\left(R^{n}, 2 e^{n+\frac{1}{2}}\right)= & \left(e_{t}^{n}, 2 e^{n+\frac{1}{2}}\right)-\left(e_{t x \bar{x}}^{n}, 2 e^{n+\frac{1}{2}}\right)+\left(I_{1}, 2 e^{n+\frac{1}{2}}\right)+\left(I_{2}, 2 e^{n+\frac{1}{2}}\right) \\
& +\left(I_{3}, 2 e^{n+\frac{1}{2}}\right)+\left(I_{4}, 2 e^{n+\frac{1}{2}}\right)+\left(I_{5}, 2 e^{n+\frac{1}{2}}\right) \\
= & \frac{1}{\tau}\left(\left\|e^{n+1}\right\|^{2}-\left\|e^{n}\right\|^{2}\right)-\frac{1}{\tau}\left(\left\|e_{x}^{n+1}\right\|^{2}-\left\|e_{x}^{n}\right\|^{2}\right)+\left(I_{1}, 2 e^{n+\frac{1}{2}}\right)+\left(I_{2}, 2 e^{n+\frac{1}{2}}\right) \\
& +\left(I_{3}, 2 e^{n+\frac{1}{2}}\right)+\left(I_{4}, 2 e^{n+\frac{1}{2}}\right)+\left(I_{5}, 2 e^{n+\frac{1}{2}}\right)
\end{aligned}
$$

Taking Schwartz inequality, it follows that

$$
\left(R^{n}, 2 e^{n+\frac{1}{2}}\right) \leq 2\left\|R^{n}\right\| \cdot\left\|e^{n+\frac{1}{2}}\right\| \leq\left\|R^{n}\right\|^{2}+2\left(\left\|e^{n+1}\right\|^{2}+\left\|e^{n}\right\|^{2}\right)
$$

From the above discussion, we have

$$
\begin{aligned}
& \left\|e^{n+1}\right\|^{2}-\left\|e^{n}\right\|^{2}+\left\|e_{x}^{n+1}\right\|^{2}-\left\|e_{x}^{n}\right\|^{2} \\
& \leq C \tau\left\|R^{n}\right\|^{2}+C \tau\left(\left\|e^{n+1}\right\|^{2}+\left\|e^{n}\right\|^{2}+\left\|e_{x}^{n+1}\right\|^{2}+\left\|e_{x}^{n}\right\|^{2}\right)
\end{aligned}
$$

Let $\varphi_{n}=\left\|e^{n}\right\|^{2}+\left\|e_{x}^{n}\right\|^{2}$, then (4.3) becomes to

$$
\varphi_{n+1}-\varphi_{n} \leq C \tau\left(\varphi_{n+1}+\varphi_{n}\right)+C \tau\left\|R^{n}\right\|^{2}
$$

Hence we have

$$
\begin{gathered}
\varphi_{n}-\varphi_{n-1} \leq C \tau\left(\varphi_{n}+\varphi_{n-1}\right)+C \tau\left\|R^{n-1}\right\|^{2} \\
\varphi_{n-1}-\varphi_{n-2} \leq C \tau\left(\varphi_{n-1}+\varphi_{n-2}\right)+C \tau\left\|R^{n-2}\right\|^{2} \\
\vdots \\
\varphi_{1}-\varphi_{0} \leq C \tau\left(\varphi_{1}+\varphi_{0}\right)+C \tau\left\|R^{0}\right\|^{2}
\end{gathered}
$$

From the above inequalities, one has

$$
\varphi_{n} \leq \varphi_{0}+C \tau \sum_{i=0}^{n-1} \varphi_{i}+C \tau \sum_{i=0}^{n-1}\left\|R^{i}\right\|^{2}
$$

where $k \sum_{i=0}^{n-1}\left\|R^{i}\right\|^{2} \leq n \tau \max _{0 \leq i \leq n-1}\left\|R^{i}\right\|^{2} \leq T O\left(\tau^{2}+h^{2}\right)^{2}$.

Since $\varphi_{0}=0$, then $\varphi_{n} \leq C \tau \sum_{i=0}^{n-1} \varphi_{i}+C T O\left(\tau^{2}+h^{2}\right)^{2}$. From discrete Gronwall inequality, $\varphi_{n} \leq O\left(\tau^{2}+h^{2}\right)^{2}$, that is

$$
\left\|e_{x}^{n}\right\|^{2}+\left\|e^{n}\right\|^{2} \leq O\left(\tau^{2}+h^{2}\right)^{2}
$$

Then we have

$$
\left\|e^{n}\right\| \leq O\left(\tau^{2}+h^{2}\right),\left\|e_{x}^{n}\right\| \leq O\left(\tau^{2}+h^{2}\right)
$$

From Theorem 3.2, we assert

$$
\left\|e_{x}^{n}\right\|_{\infty} \leq O\left(\tau^{2}+h^{2}\right)
$$


We complete the proof of Theorem 4.2.

Theorem 4.3. The solution of the difference scheme (3.1)-(3.3) is stable in $L_{\infty}$.

Proof. Assume that $u_{j}^{n}$ is the solution of the difference scheme (3.1)-(3.3), $U_{j}^{n}$ is the solution of original differential Equations (1.6)-(1.8). We can easily obtain that $e_{j}^{n}=U_{j}^{n}-u_{j}^{n}$.

Then from Theorem 4.2, the following inequality holds true

$$
\left\|e^{n}\right\|^{2} \leq C\left\|U_{0}-u_{0}\right\|^{2}
$$

Thus, we complete the proof of Theorem 4.3 .

\section{Conclusion}

In this paper, we give a difference scheme for the generalized Novikov equation. In Section 2, we give some preparation knowledge. In Section 3, we propose a conservative finite difference scheme for the generalized Novikov equation and use Brouwer fixed point theorem to obtain the existence of the solution for the corresponding difference equation. In Section 4, we prove the convergence and stability of the solution by using the discrete energy method.

\section{Acknowledgements}

This research is supported by the National Nature Science Foundation of China (Grant Nos. 11501253, 11571141 and 11571140), and the Nature Science Foundation of Jiangsu Province (Grant No. BK20140525), and the Advanced talent of Jiangsu University (Grant Nos. 14JDG070, 15JDG079).

\section{References}

[1] Camassa, R. and Holm, D.D. (1933) An Integrable Shallow Water Equation with Peaked Solitons. Physical Review Letters, 71, 1661-1664. https://doi.org/10.1103/PhysRevLett.71.1661

[2] Constantin, A. and Lannes, D. (2009) The Hydrodynamical Relevance of the Camassa-Holmand Degasperis-Procesi Equations. Archive for Rational Mechanics and Analysis, 192, 165-186. https://doi.org/10.1007/s00205-008-0128-2

[3] Johnson, R.S. (2002) Camassa-Holm, Korteweg-de Vries and Related Models for Water Waves. Journal of Fluid Mechanics, 455, 63-82. https://doi.org/10.1017/S0022112001007224

[4] Fuchssteiner, B. and Fokas, A.S. (1981/1982) Symplectic Structures, Their Bäcklund Transformations and Hereditary Symmetries. Physica D, 4, 47-66.

[5] Olver, P.J. and Rosenau, P. (1996) Tri-Hamiltonian Duality between Solitons and Solitary-Wave Solutions Having Compact Support. Physical Review E, 53, 1900-1906. https://doi.org/10.1103/PhysRevE.53.1900

[6] Fuchssteiner, B. (1996) Some Tricks from the Symmetry-Toolbox for Nonlinear Equations: Generalizations of the Camassa-Holm Equation. Physica D, 95, 229-243. https://doi.org/10.1016/0167-2789(96)00048-6

[7] Cao, C.S., Holm, D.D. and Titi, E.S. (2004) Traveling Wave Solutions for a Class of One-Dimensional Nonlinear Shallow Water Wave Models. Journal of Dynamics 
and Differential Equations, 16, 167-178.

[8] Kouranbaeva, S. (1999) The Camassa-Holm Equation as a Geodesic Flow on the Diffeomorphism Group. Journal of Mathematical Physics, 40, 857-868.

https://doi.org/10.1063/1.532690

[9] Constantin, A. and Kolev, B. (2002) On the Geometric Approach to the Motion of Inertial Mechanical Systems. Journal of Physics A, 35, R51-R79. https://doi.org/10.1088/0305-4470/35/32/201

[10] Chou, K.S. and Qu, C.Z. (2002) Integrable Equations Arising from Motions of Plane Curves I. Physica D, 162, 9-33. https://doi.org/10.1016/S0167-2789(01)00364-5

[11] Constantin, A. (2000) Existence of Permanent and Breaking Waves for a Shallow Water Equation: A Geometric Approach. Annales de P Institut Fourier, 50, 321-362. https://doi.org/10.5802/aif.1757

[12] Constantin, A. and Escher, J. (1998) Wave Breaking for Nonlinear Nonlocal Shallow Water Equations. Acta Mathematica, 181, 229-243. https://doi.org/10.1007/BF02392586

[13] Constantin, A. and Escher, J. (1998) Global Existence and Blow-Up for a Shallow Water Euation. Annali della Scuola Normale Superiore di Pisa, 26, 303-328.

[14] Li, Y.A. and Olver, P. (2000) Well-Posedness and Blow-Up Solutions for an Integrable Nonlinearly Dispersive Model Wave Equation. Journal of Differential Equations, 162, 27-63. https://doi.org/10.1006/jdeq.1999.3683

[15] Constantin, A. and Molinet, L. (2001) Orbital Stability of Solitary Waves for a Shallow Water Equation. Physica D, 157, 75-89.

[16] Constantin, A. and Strauss, W. (2000) Stability of Peakons. Communications on Pure and Applied Mathematics, 53, 603-610. https://doi.org/10.1002/(SICI)1097-0312(200005)53:5<603::AID-CPA3>3.0.CO;2-L

[17] Degasperis, A. and Procesi, M. (1999) Asymptotic Integrability. In: Degasperis, A., et al., Eds., Symmetry and Perturbation Theory, World Scientific, Singapore, 23-37.

[18] Liu, Y. and Yin, Z. (2006) Global Existence and Blow-Up Phenomena for the Degasperis-Procesi Equation. Communications in Mathematical Physics, 267, 801-820. https://doi.org/10.1007/s00220-006-0082-5

[19] Escher, J., Liu, Y. and Yin, Z. (2006) Global Weak Solutions and Blow-Up Structure for the Degasperis-Procesi Equation. Journal of Functional Analysis, 241, 457-485.

[20] Escher, J., Liu, Y. and Yin, Z. (2007) Shock Waves and Blow-Up Phenomena for the Periodic Degasperis-Procesi Equation. Indiana University Mathematics Journal, 56, 87-117. https://doi.org/10.1512/iumj.2007.56.3040

[21] Lin, Z.W. and Liu, Y. (2009) Stability of Peakons for the Degasperis-Procesi Equation. Communications on Pure and Applied Mathematics, 62, 125-146.

[22] Lundmark, H. (2007) Formation and Dynamics of Shock Waves in the Degasperis-Procesi Equation. Journal of Nonlinear Science, 17, 169-198.

https://doi.org/10.1007/s00332-006-0803-3

[23] Fu, Y., Gui, G.L., Liu, Y. and Qu, C.Z. (2013) On the Cauchy Problem for the Integrable Camassa-Holm Type Equation with Cubic Nonlinearity. Journal of Differential Equations, 255, 1905-1938.

[24] Gui, G.L., Liu, Y., Olver, P. and Qu, C.Z. (2013) Wave-Breaking and Peakons for a Modified Camassa-Holm Equation. Communications in Mathematical Physics, 319, 731-759. https://doi.org/10.1007/s00220-012-1566-0

[25] Qu, C.Z., Liu, X.C. and Liu, Y. (2013) Stability of Peakons for an Integrable Mod- 
ified Camassa-Holm Equation. Communications in Mathematical Physics, 322, 967-997. https://doi.org/10.1007/s00220-013-1749-3

[26] Qu, C.Z., Zhang, Y., Liu, X.C. and Liu, Y. (2014) Orbital Stability of Periodic Peakons to a Generalized Camassa-Holm Equation. Archive for Rational Mechanics and Analysis, 211, 593-617. https://doi.org/10.1007/s00205-013-0672-2

[27] Novikov, V. (2009) Generalizations of the Camassa-Holm Equation. Journal of Physics A, 42, Article ID: 342002.

[28] Hone, A.N., Lundmark, H. and Szmigielski, J. (2009) Explicit Multipeakon Solutions of Novikov's Cubically Nonlinear Integrable Camassa-Holm Type Equation. Dynamics of Partial Differential Equations, 6, 253-289. https://doi.org/10.4310/DPDE.2009.v6.n3.a3

[29] Hone, A.N. and Wang, J.P. (2008) Integrable Peakon Equations with Cubic Nonlinearity. Journal of Physics A, 41, Article ID: 372002.

[30] Tiglay, F. (2011) The Periodic Cauchy Problem for Novikov's Equation. International Mathematics Research Notices, 2011, 4633-4648.

[31] Wu, X. and Yin, Z. (2011) Global Weak Solutions for the Novikov Equation. Journal of Physics A, 44, Article ID: 055202.

[32] Fokas, A.S. (1995) The Korteweg-de Vries Equation and Beyond. Acta Applicandae Mathematicae, 39, 295-305. https://doi.org/10.1007/BF00994638

[33] Qiao, Z., Xia, B. and Li, J.B. (2012) Integrable System with Peakon, Weak Kink, and Kink-Peakon Interactional Solutions. http://xxx.lanl.gov/abs/1205.2028

[34] Zhou, Y. (1990) Application of Discrete Functional Analysis to the Finite Difference Methods. International Academic Publishers, Beijing.

\section{Submit or recommend next manuscript to SCIRP and we will provide best} service for you:

Accepting pre-submission inquiries through Email, Facebook, LinkedIn, Twitter, etc. A wide selection of journals (inclusive of 9 subjects, more than 200 journals)

Providing 24-hour high-quality service

User-friendly online submission system

Fair and swift peer-review system

Efficient typesetting and proofreading procedure

Display of the result of downloads and visits, as well as the number of cited articles

Maximum dissemination of your research work

Submit your manuscript at: http://papersubmission.scirp.org/

Or contact jamp@scirp.org 